\title{
Optical Polarizability of Zigzag Single-Walled Carbon Nanotubes Fullerene-Capped at One End and Covalently Bonded with Benzene Rings at the Other End
}

\author{
O. V. Ogloblya, ${ }^{1}$ D. Hui, ${ }^{2}$ Y. M. Strzhemechny, ${ }^{3}$ and Yu. I. Prylutskyy ${ }^{1}$ \\ ${ }^{1}$ Department of Biophysics, The Faculty of Biology, National Taras Shevchenko University of Kyiv, Volodymyrska Street 64, \\ 01033 Kyiv, Ukraine \\ ${ }^{2}$ Department of Mechanical Engineering, University of New Orleans, New Orleans, LA 70148, USA \\ ${ }^{3}$ Department of Physics and Astronomy, Texas Christian University, TCU Box 298840, Fort Worth, TX 76129, USA
}

Received 26 March 2007; Accepted 31 July 2007

Recommended by S. Mathur

\begin{abstract}
We report on the results of numerical simulations for the linear optical polarizability of single-walled zigzag $(9,0)$ carbon nanotubes with modified ends. The nanotubes of a variable length are fullerene-capped at one end and covalently bonded to a hydrophobic cluster of nine benzene rings at the other end. We investigate electronic and optical properties of such structures within a framework of the Su-Schrieffer-Heeger model. We demonstrated that the localized states in this system exhibit nonlinear characteristics of excited states. The nanotubes have a strongly oscillating dependence of their optical polarizability on the energy of incident light. Spectral features of the optical polarizability drop in intensity and shift towards higher energies with a decrease in the length of a nanotube or upon fullerene-uncapping. The length dependence is similar for the nanotubes without benzene rings, capped either at one or both ends. Potential applications are suggested for hydrophobic pollutant control in liquid-purification systems.
\end{abstract}

Copyright (c) 2007 O. V. Ogloblya et al. This is an open access article distributed under the Creative Commons Attribution License, which permits unrestricted use, distribution, and reproduction in any medium, provided the original work is properly cited.

\section{INTRODUCTION}

Properties of single-walled carbon nanotubes (SWCNTs) remain at the center of vigorous research efforts by numerous theoretical and experimental groups. Reports are abundant on the fabrication of significant quantities of SWCNTs few nanometers in diameter of a narrow size distribution, uncapped or fullerene-capped at the ends employing various transition and lanthanide metal-based catalysts. Furthermore, additional interest is drawn to the properties of nanotubes functionalized by placing different organic radicals at the ends. One of such configurations suggested here could be formed by fullerene-capping at one end of the nanotube and bonding by a hydrophobic cluster of nine benzene rings at the other end. Such structures could be utilized in liquid purification applications by absorbing low-molecular hydrophobic pollutants through the hydrophobic clusters into the tubes.

Obviously, optical properties of these systems could serve as a sensitive probe of the efficiency of such purification scheme. This offers a sound rationale to study optical polar- izability of uncapped and fullerene-capped nanotubes functionalized by benzene rings at the opposite end.

In our studies we utilize the Su-Schrieffer-Heeger (SSH) model [1-4], which accounts for the Coulomb interaction and modifies accordingly the Peierls-Hubbard model. In the SSH model, the electron-phonon interaction is included and causes the Peierls distortions, which in the case of nanotubes result in dimerization.

There are recent reports (see, e.g., [5]) showing that the conjugated $\pi$-electron organic systems are potentially important in photonic applications due to their large nonlinear optical (NLO) response. However, such polymeric materials exhibit a residual infrared absorption, which is caused by $\mathrm{C}-\mathrm{H}$ stretching vibrations and is detrimental in infrared applications. Since fullerenes and nanotubes are free from such phenomena, this makes them an appealing NLO material. Because of this, second-order hyperpolarizabilities of fullerens and nanotubes generated substantial research effort $[6,7]$. Nonetheless, linear polarizability of nanotubes, and especially nanotubes with different end configurations, such as attached organic radicals, remains practically unaddressed. 


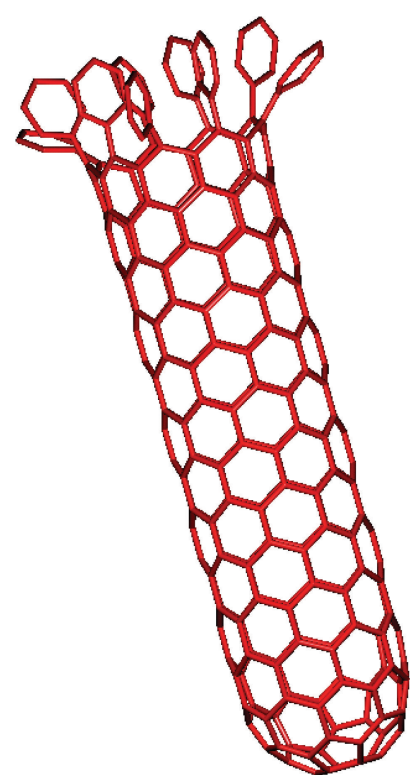

FIgUre 1: Configurational geometry of a $(9,0)$-SWCNT, with a length of five translational periods, fullerene-capped at one end and covalently bonded with nine benzene rings at the other end.

\section{RESULTS AND DISCUSSION}

We consider a $(9,0)$ SWCNT with a diameter of about $0.7 \mathrm{~nm}$, a variable length, and three different end configurations: a fullerene cap, an array of benzene rings, and uncapped. Therefore, one possible configuration is capping by a half of a fullerene $\mathrm{C}_{60}$ molecule at one end and covalent bonding with nine benzene rings at the other, as shown in Figure 1. Other possibilities include fullerene capping at both ends or fullerene capping at one end with the other end uncapped. In our studies we investigate $(9,0)$-SWCNT clusters containing 4, 5, and 6 translation periods.

In the simulations of electronic and optical properties, the Su-Schrieffer-Heeger [1-4] model is employed.

The eigenvalues $\varepsilon_{k s}$ and the eigenfunctions $\psi_{k s}$ are found from the Schrödinger equation for the $\pi$-electrons [4]:

$$
\begin{aligned}
\varepsilon_{k s} \psi_{k s}(i)= & -\sum_{\langle i, j\rangle}\left(1+\xi y_{i j}+v \tau_{i j, s}\right) \psi_{k s}(j) \\
& +\left(u \rho_{i, s}+\nu \sum_{\langle i, j\rangle, s^{\prime}} \rho_{i, s^{\prime}}\right) \psi_{k s}(i) .
\end{aligned}
$$

Here, $\rho_{i s}=\sum_{k}^{\prime} \psi_{k s}(i)^{2}, \quad \tau_{i j, s}=\sum_{k}^{\prime} \psi_{k s}(i) \psi_{k s}(j)$ and priming of the sum symbol indicates summation over the populated levels only. Equation (1) is a modified form of a standard one $[3,4]$, which we obtained by introducing parameters $u=U / t, v=V / t$, and $\xi=\alpha^{2} / K t$. This leads to the energy units being measured in $t$ (hopping integral between two $\pi$-type bonds). Our computations are not sensitive to the values of $t$, however, at the end the value $t=2.5, \mathrm{eV}$ is used.
From the constraint equation $\sum_{\langle i j\rangle} y_{i j}=0$, it is possible to obtain the self-consistency equation for $y_{i j}$ :

$$
y_{i j}=\sum_{s} \tau_{i j, s}-\Delta y, \quad \Delta y=\frac{1}{N_{\pi}} \sum_{\langle i j, s\rangle} \tau_{i j, s .}
$$

Here, $N_{\pi}$ is the number of $\pi$-type bonds. The following numerical values are chosen for the parameters in our numerical calculations: $\xi=0.32, U / t=1.0, V / t=0.6$.

To calculate the linear optical polarizability, we used the sum-over-state approach $[8,9]$

$$
\alpha(E)=\sum_{\substack{n \in \text { occ. } \\ p \in \text { unocc. }}} \mu_{n p} \mu_{p n}\left(\frac{2 \varepsilon_{p n}\left(\varepsilon_{p n}^{2}-E^{2}\right)}{\left(\varepsilon_{p n}^{2}-E^{2}\right)^{2}+\Gamma^{2} E^{2}}\right) .
$$

Here, $\mu_{n p}=\sum_{j, s} \psi_{n s}(j)\left(-e r_{j}\right) \psi_{p s}(j)$ is the dipole moment, whereas $\Gamma=10 \mathrm{meV}$, which accounts for the radiationwidening of the levels. We are interested in the spatially averaged optical polarizability, which can be written as $\alpha=$ $\left(\alpha_{x x}+\alpha_{y y}+\alpha_{z z}\right) / 3$.

Computational method described above was verified first to calculate $\alpha(0)$, the static linear polarizability of $\mathrm{C}_{60}$. We obtained a value of $82 \AA^{3}$ for $\alpha(0)$, which was in good agreement with the available experimental and theoretical data [10-12].

In Figure 2(c), the dashed line represents the calculated optical polarizability for a tube structure shown in Figure 1. Overall, Figure 2 summarizes calculated polarizabilities for tube structures of three different end configurations and three different lengths.

One can see from Figure 2 that the peaks of optical polarizability are shifting towards smaller energies for structures with greater tube length. Moreover, the intensities of the first peak of the optical polarizability as well as the values of the static linear polarizability increase with the increasing tube length. Presence of a second fullerene cap in Figures 2(a) and 2(b) also shifts the peaks towards smaller energies, which can be explained by the increase of the effective dimensionality of the system. Presence of the benzene array at the end shifts the optical polarizability peaks towards smaller energies, although this shift is not as significant as in the case of fullerene cap configuration at the end. The effect of caps is greater for the structures of smaller length. As we can see, in the case of length corresponding to four translational periods (two adjacent rings from hexagons is a unit cell) of a zigzag nanotube (Figure 2, dotted line), the spectral shapes are substantially different for Figure 2(b) versus Figure 2(a) or Figure 2(c). Therefore, SWCNT of such length when capped at both ends could be easily distinguished.

\section{CONCLUSION}

Numerically calculated spectra of optical polarizability for SWCNT-fullerene-capped and covalently bonded with benzene rings-have a strong oscillatory dependence on the incident light energy resulting from a complex electronic structure of such systems. Decrease in length and uncapping of nanotubes' ends shift the spectral features towards higher energies and reduce their intensity. The polarizability spectra of isolated capped $(9,0)$ SWCNTs are in good agreement with the first-principle calculations [12]. 


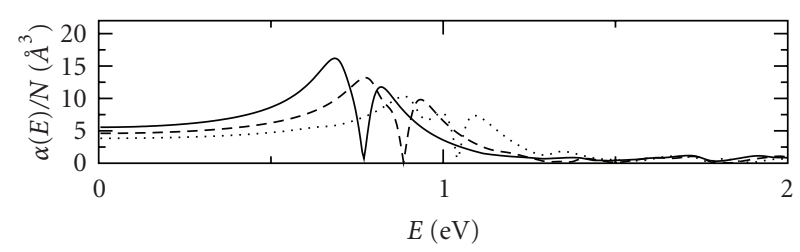

(a)

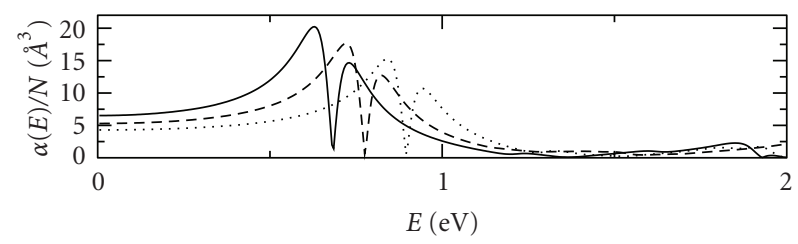

(b)

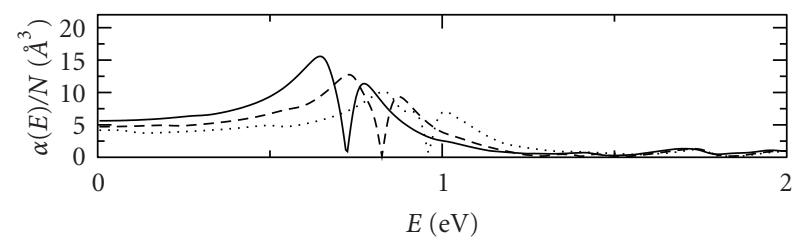

(c)

FIGURE 2: Optical polarizability of a $(9,0)$ zigzag SWCNT: (a) fullerene-capped only at one end, (b) fullerene-capped at both ends, (c) fullerene-capped at one end and covalently bonded to nine benzene rings at the other end. Polarizabilities are calculated for SWCNT with nanotube fragments of three different lengths: 4 (dotted line), 5 (dashed line), and 6 (solid line) translational periods.

We suggest that optical spectroscopy can be used to quickly determine the quantities of empty (opened at one end) and filled (closed at both ends by a cap or shielded by benzene rings) SWCNTs in liquids, by observing their spectral differences. Such structures could be utilized in liquid purification applications by absorbing low-molecular hydrophobic pollutants into the tubes.

We believe that optical polarizability experiments can be readily performed on the SWCNT objects described above. Moreover, studies of structural properties of a relatively simple system discussed in this paper should be instrumental, in our view, when studying more complex structures consisting of smaller "building blocks" akin to the ones reported here.

\section{REFERENCES}

[1] A. J. Heeger, S. Kivelson, J. R. Schrieffer, and W.-P. Su, "Solitons in conducting polymers," Reviews of Modern Physics, vol. 60, no. 3, pp. 781-850, 1988.

[2] X. Wan, J. Dong, and D. Y. Xing, "Optical properties of carbon nanotubes," Physical Review B, vol. 58, no. 11, pp. 6756-6759, 1998.

[3] J. Yu and W.-P. Su, "Nonlinear optical susceptibilities of manyelectron systems," Physical Review B, vol. 44, no. 24, pp. 13315-13318, 1991.

[4] J. Ma and R.-K. Yuan, "Electronic and optical properties of finite zigzag carbon nanotubes with and without Coulomb interaction," Physical Review B, vol. 57, no. 15, pp. 9343-9348, 1998.
[5] D. Lu, B. Marten, M. Ringnalda, R. A. Friesner, and W. A. Goddard III, "Saturation of the second hyperpolarizability for polyacetylenes," Chemical Physics Letters, vol. 257, no. 3-4, pp. 224-228, 1996.

[6] R.-H. Xie and J. Jiang, "Nonlinear optical properties of armchair nanotube," Applied Physics Letters, vol. 71, no. 8, pp. 1029-1031, 1997.

[7] R.-H. Xie and J. Jiang, "Theory of nonlinear optical properties of $\mathrm{C}_{60}$-derived nanotubes," Journal of Applied Physics, vol. 83, no. 6, pp. 3001-3007, 1998.

[8] O. V. Ogloblya and G. M. Kuznetsova, "Peculiarities of the optical polarizability of single-walled zigzag carbon nanotube with capped and tapered ends," in Proceedings of the NATO Advanced Study Institute of Carbon Nanotubes: From Basic Research to Nanotechnology, pp. 173-174, Sozopol, Bulgaria, May 2005.

[9] Yu. Prylutskyy, O. Ogloblya, E. Buzaneva, A. Gorchinskiy, P. Eklund, and P. Scharff, "Optical properties of single-walled carbon nanotubes," Functional Materials, vol. 7, pp. 652-654, 2000.

[10] J.-I. Iwata, K. Yabana, and G. F. Bertsch, "Real-space computation of dynamic hyperpolarizabilities," Journal of Chemical Physics, vol. 115, no. 19, pp. 8773-8783, 2001.

[11] A. Ballard, K. Bonin, and J. Louderback, "Absolute measurement of the optical polarizability of $\mathrm{C}_{60}$," Journal of Chemical Physics, vol. 113, no. 14, pp. 5732-5735, 2000.

[12] M. Bianchetti, P. F. Buonsante, F. Ginelli, H. E. Roman, R. A. Broglia, and F. Alasia, "Ab-initio study of the electromagnetic response and polarizability properties of carbon chains," Physics Reports, vol. 357, no. 6, pp. 459-513, 2002. 

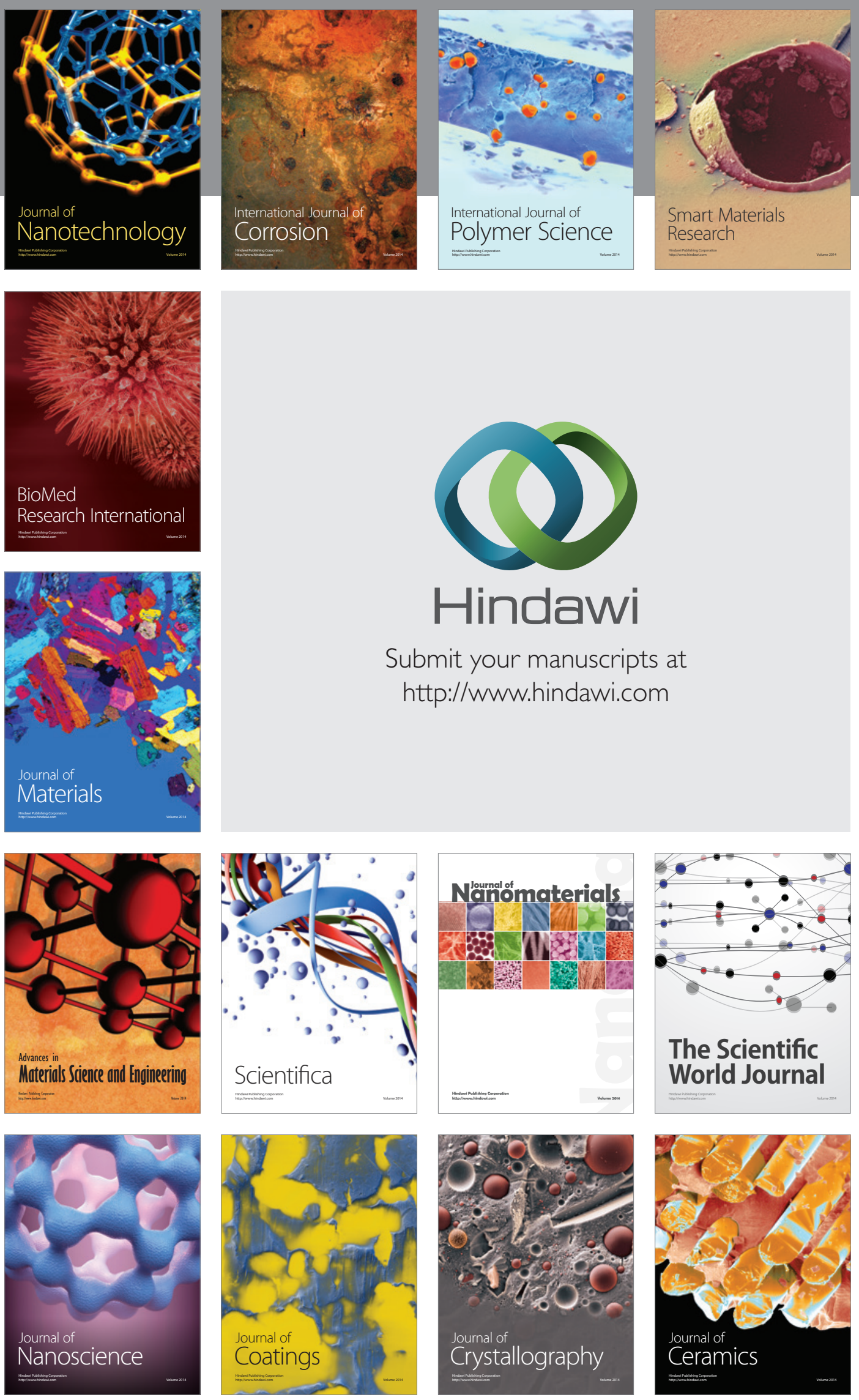

The Scientific World Journal

Submit your manuscripts at

http://www.hindawi.com

\section{World Journal}

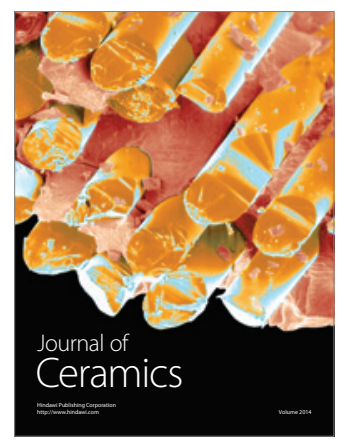

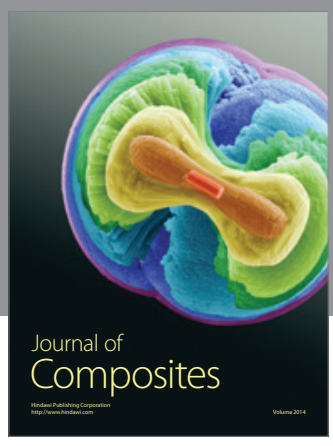
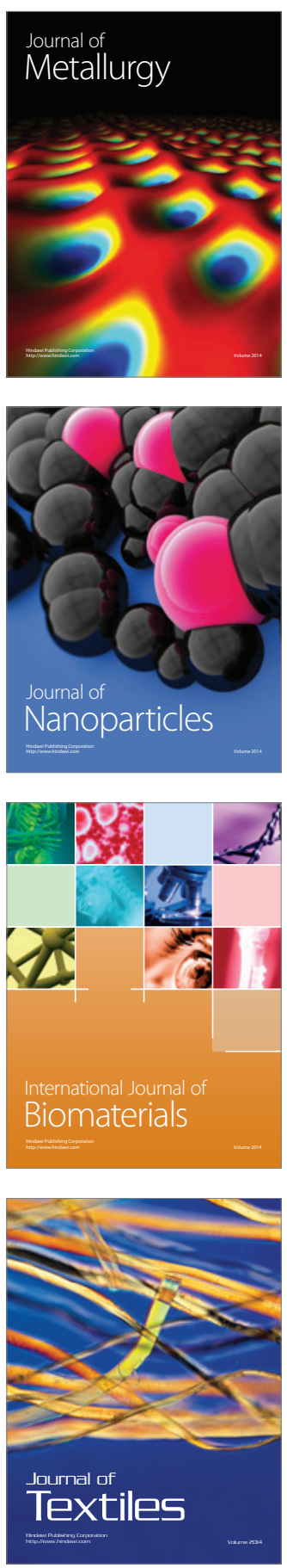